\title{
Radiological and Histological Evaluation of Regenos $®$ Which Implanted in Human Radial Fracture: A Clinical Case Report
}

\author{
Masashi Iwasashi MD, PhD ${ }^{1, a}$, Toshiki Muramatsu MD ${ }^{1, b}$ \\ and Masataka Sakane MD, PhD ${ }^{2, \mathrm{c}}$ \\ ${ }^{1}$ Department of Orthopaedic Surgery, Showa General Hospital, 2-450 Tenjin, Kodaira, Tokyo, \\ 187-8510, JAPAN \\ ${ }^{2}$ Department of Orthopaedic Surgery, University of Tsukuba, 1-1-1 Tennodai, Tsukuba, Ibaraki \\ 305-8575, Japan \\ aiwasashi-m@hotmail.co.jp, ${ }^{\mathrm{b}}$ muramatu@dg7.so-net.ne.jp, 'sakane-m@md.tsukuba.ac.jp
}

Keywords: Regenos $®$, unidirectional porous hydroxyapatite (UDPHAp), clinical application, radiological evaluation, histological evaluation

Abstract Regenos ${ }^{\circledR}$ is a unidirectional porous hydroxyapatite (UDPHAp) bone substitutes, and the most distinctive feature of UDPHAp is its interconnected porous structure. We used Regenos ${ }^{\circledR}$ for the bone defect while distal radius fracture surgery with metal fixation implant. At six months post-operation, CT scans revealed the Regenos ${ }^{\circledR}$ implant was uniformly composed of cortical bone adjacent to the trabecular bone. At one-year postoperatively, we collected a sample of the implanted Regenos ${ }^{\circledR}$ while metal fixation plate removal surgery for histological evaluation. Regenos ${ }^{\circledR}$ implant sample revealed the presence of ossified bone stained green with Villanueva Goldner stain. Our findings demonstrate that Regenos ${ }^{\circledR}$ is a useful bone substitution material in the clinical setting.

\section{Introduction}

Regenos ${ }^{\circledR}$ (Kuraray Medical, Inc., Tokyo, Japan) is a unidirectional porous hydroxyapatite (UDPHAp) material with $75 \%$ porosity and $99.9 \%$ purity. The most distinctive feature of UDPHAp is its interconnected porous structure, consisting of unidirectional oval pores (approximately 100-300 $\mu \mathrm{m}$ in the longest diameter) that completely penetrate through the material (Fig. 1). The pore size and microstructure of Regenos ${ }^{\circledR}$ facilitate the invasion of cells and fluids necessary for osteogenesis, as demonstrated by the immediate permeation of blood through the material by capillary action when the UDPHAp. We previously reported the favorable osteogenesis within UDPHAp implants in the femoral bone marrow of rabbits 0. While UDPHAp was authorized by Ministry of Health, Labour and Welfare in 2009 as Regenos ${ }^{\circledR}$; there are a few clinical reports about radiological and histological evaluation.

During follow-up surgery to remove the metal used for internal fixation in the radius fracture surgery in which a Regenos ${ }^{\circledR}$ block was implanted in the bone defect, we collected a sample of the implanted material for histological evaluations.

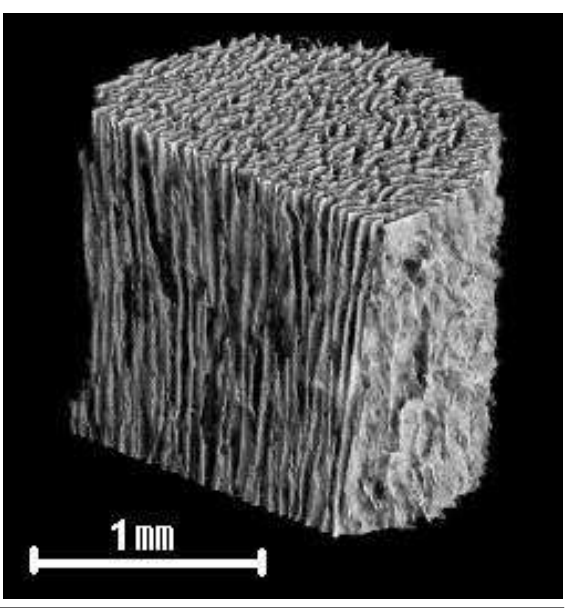

[Fig.1] 3-D micro CT image of the UDPHAp shows unidirectional pore toward the vertical direction and some interconnection toward the horizontal direction at the top. Here, we report the clinical course of fracture healing and radiological findings about the Regenos ${ }^{\circledR}$ implant, and the histopathological evaluation results.

\section{Materials and Methods}

A 57-year-old female fell and sustained a distal radius fracture. She underwent open reduction and internal fixation surgery with a APTUS ${ }^{\circledR}$ Radius 2.5 volar locking plate (Medartis AG, Switzerland). During the surgery, a $10-\mathrm{mm}$ cubic Regenos ${ }^{\circledR}$ block was shaped to fit the bone defect for 
implantation into the fracture site. After surgery, the patient was periodically examined by X-ray and given CT scans at 6 weeks and 6 months. At one-year postoperatively, the patient underwent plate removal surgery, during which time we collected a cylindrical sample (1.4 $\mathrm{mm}$ in diameter) of the implanted Regenos ${ }^{\circledR}$ using a cannulated drill. The excised sample was fixed in $4 \%$ formalin, embedded in ethyl methacrylate (MMA) resin, sectioned at $6-\mu$ m thickness using a LEICA RM2255 microtome. The sections were stained with Villanueva Goldner (VG) for observation under a light microscope. The patient provided written informed consent for the collection of samples and subsequent analyses. The study was approved by the local ethics committee of Showa general hospital.

\section{Results}

The fracture patient followed a favorable post-operative clinical course and started wrist excursion training after one week of external fixation. During the 19-month follow-up period, no complications, including infection, were encountered. The patient's wrist range of motion and grip strength were $82 \%$ and $86 \%$, respectively, compared with the those of the unaffected wrist (dominant hand), and the patient reported no impairment of daily activities.

At 6 weeks post-operation, signs of bone healing at the fracture site were detected in X-ray images (Fig.2b). In addition, CT scans revealed continuity between the Regenos ${ }^{\circledR}$ implant and the surrounding bone. And also, a fixation screw passed through the Regenos ${ }^{\circledR}$ implant, no associated damage was detected (Fig.3a). At six months post-operation, the Regenos ${ }^{\circledR}$ implant was uniformly composed of cortical bone adjacent to the trabecular bone (Fig.2b). However, the post-operative gap between the Regenos ${ }^{\circledR}$ implant and the surrounding bone at the fracture site remained until the final evaluation performed at 19 months post-operation (Fig.2c, 3a, 3b).
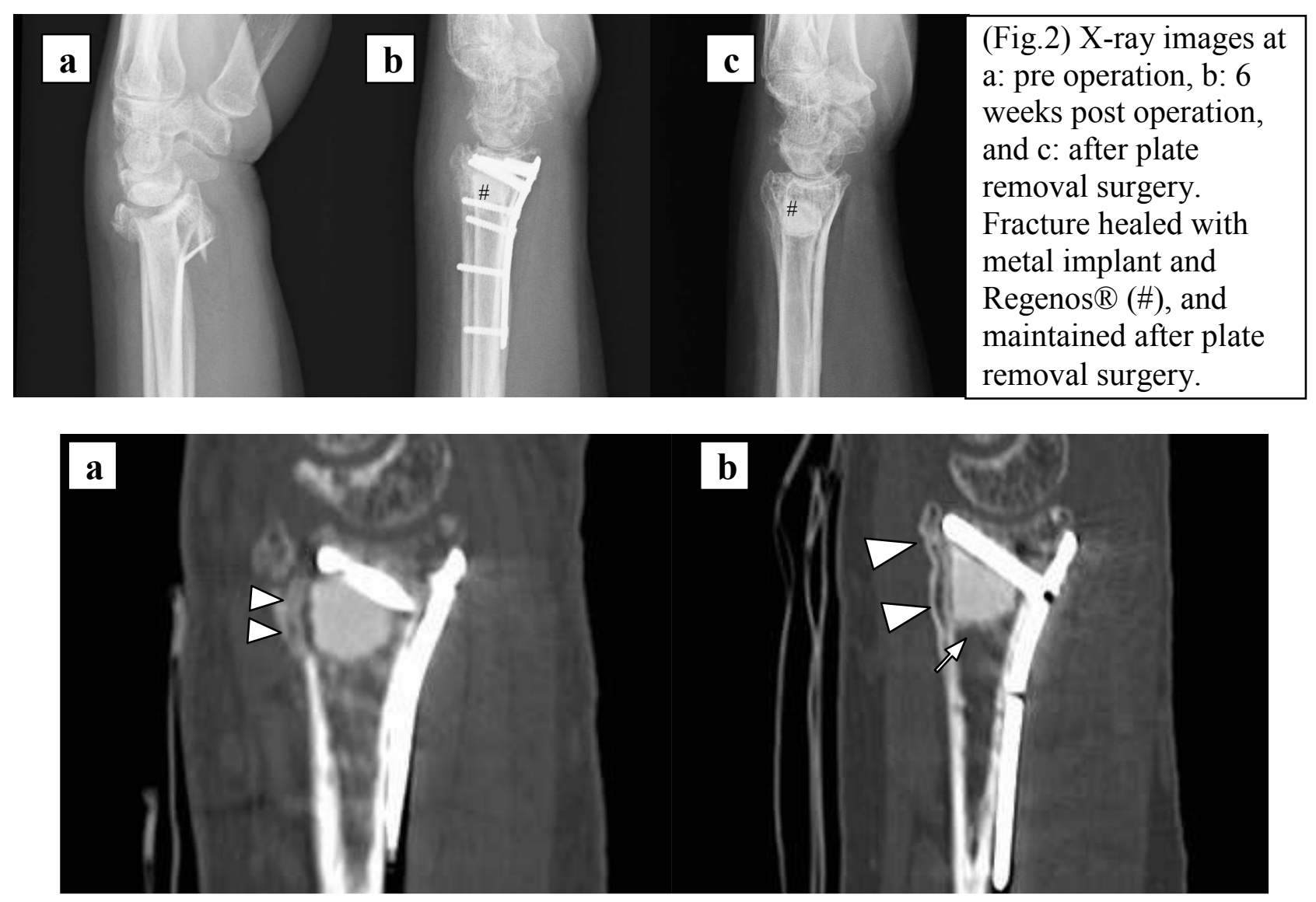

(Fig.3) CT scans at a: 6 weeks and b: 6 months post operation. At six months post-operation, the Regenos ${ }^{\circledR}$ implant was uniformly composed of cortical bone adjacent to the trabecular bone (arrow). Gap between the Regenos ${ }^{\circledR}$ implant and the surrounding bone at the fracture site remained (arrow head). 
Histological examination of the Regenos ${ }^{\circledR}$ implant sample revealed the presence of ossified bone stained green with VG was lengthening (Fig.4), a finding that is consistent with the microstructure of Regenos ${ }^{\circledR}$. No osteoblasts or osteoclasts were detected within this sample.

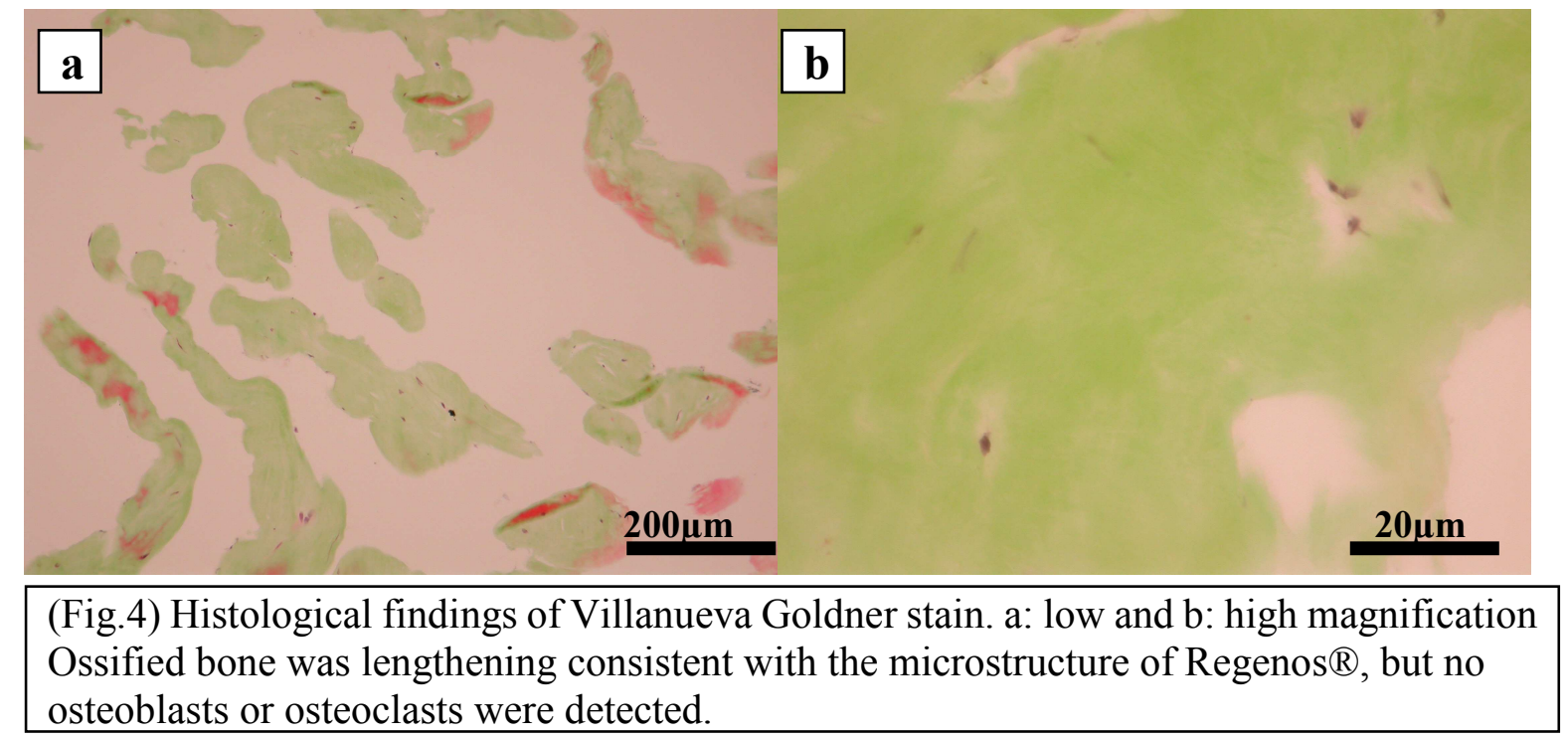

\section{Discussions}

The unidirectional interconnected porous structure of Regenos ${ }^{\circledR}$ has several advantages when used as a bone substitute material. First, the pore size $(100-300 \mu \mathrm{m})$ and interconnectivity of this material facilitate invasion of the cells and growth factors that are necessary for osteogenesis to proceed. Karageorgiou et al.[3] reported that a $100-300-\mu \mathrm{m}$ pore size is suitable for promoting angiogenesis. Furthermore, the unidirectional pore structure of Regenos ${ }^{\circledR}$ permits the rapid movement of blood through the material by capillary action, a property that not only promotes cell invasion, but also the immediate supply of the necessary fluid components necessary for the growth and differentiation of cells that have penetrated the material.

Second, the unidirectional interconnected porous structure of Regenos ${ }^{\circledR}$ is also dynamically advantageous in the clinical setting as it increases the compression strength of the material in the direction of the pores. Furthermore, as osteogenesis occurs within the Regenos ${ }^{\circledR}$ material, artificial bone and autogenous bone are unified, increasing the compression strength of the implantation site. Our previous experiments showed the occurrence of osteogenesis inside UDPHAp implanted in the rabbit femoral cavity, with compression strength increasing 1.5- and 3.4-fold at 6 and 12 weeks after implantation, respectively, compared to that of before implantation 0 .

In the present clinical case, Regenos ${ }^{\circledR}$ was used to repair a bone defect that resulted after a wrist fracture, which is common in middle-aged and elderly women due to the underlying condition of osteoporosis. At the time of fracture, cancellous bone is compressed, often resulting in bone defects after fracture reduction. Although volar locking plates are typically used for fixation in fracture surgery, the repair of severe bone defects often requires bone implantation or bone substitution. The compression strength of Regenos ${ }^{\circledR}$ in the direction of the interconnected pores is $13 \mathrm{MPa}$, which is similar to that of cancellous bone and sufficiently strong to function as a substitute for non-weight bearing joints. Furthermore, Regenos ${ }^{\circledR}$ block can be easily shaped to fit the bone defect site intraoperatively, the defect can buried precisely. Despite these advantages, caution is needed during the implantation of Regenos ${ }^{\circledR}$ blocks to avoid applying large amounts of force, as the unique microstructure of UDPHAp makes it susceptible to forces in the vertical direction for interconnected porous. 
During the 19-month post-operative period, the clinical course of fracture healing was favorable, and no complications associated with Regenos ${ }^{\circledR}$ implantation were found. Imaging evaluation showed no indication of post-implantation fractures of the Regenos ${ }^{\circledR}$. Although a fixation screw passed through the Regenos ${ }^{\circledR}$ implant, no associated damage was detected, which indicates that there is no need for avoiding the implanted material when inserting screws. Notably, trabecular bone exhibited vertical continuity with the surrounding tissue. However, caution should be taken to avoid gaps when implanting Regenos ${ }^{\circledR}$ blocks into bone, as the initial gap between the material and adjacent bone tissue remained during the course of fracture healing, resulting in fibrous union.

Histological examination revealed ossified bone but there was no evidence of active bone metabolism. This finding is not consistent with the previous results of Regenos ${ }^{\circledR}$ implantation into defect sites within the tibia of rabbit, in which active osteogenesis was detected up to 12 weeks after implantation [4]. The possible reasons for the observed differences in bone metabolism include inherent differences between species, the completion of bone repair at the fracture site at the time of evaluation in the human patient, and reduced bone metabolism associated with older age. Although it is difficult to obtain clinical specimens of implanted material from human subjects, further histological evaluation of such specimens is contribute to confirm the present findings.

\section{Conclusions}

During fracture surgery, Regenos ${ }^{\circledR}$ could be easily shaped to properly fit the defect site as a human bone substitute. Passing a screw through the implant material was not associated with damage or impaired osteogenesis. The Regenos ${ }^{\circledR}$ implant began to be united to the surrounding bone from around 6 weeks postoperatively, leading to fully healed bone within approximately three months, without material damage or fracture site dislocation. Notably, osteogenesis was detected in a sample of the implanted Regenos ${ }^{\circledR}$ block at one year after implantation. Our findings demonstrate that Regenos ${ }^{\circledR}$ is a useful bone substitution material in the clinical setting.

\section{References}

[1] M. Iwasashi, M. Sakane et al The Increase of the Mechanical Strength of Novel Unidirectional Porous Hydroxyapatite Ceramics in Vivo J. Bone Miner. Res. 22 (Suppl. 1) (2007) S262.

[2] Watanabe A, Sakane M, Funayama T, Iwasashi M, Kanamori A, Ochiai N, Novel unidirectional porous hydroxyapatite used as a bone substitute for tibial wedge osteotomy in canines, Biomaterials Research. 14(1) (2010)6-9.

[3] Karageorgiou V, Kaplan D, Porosity of 3D biomaterial scaffolds and osteogenesis, Biomaterials. 26(27) (2005)5474-91.

[4] Iwasashi M, Sakane M, Suetsugu Y, Ochiai N, Bone regeneration at cortical bone defect with unidirectional porous hydroxyapatite in vivo, Key Engineering Materials. 396-398(2009) 11-14. 